\title{
Maladaptive emotion regulation mediating the link between the recall of early affiliative memories and depressive symptomatology
}

\author{
Joana Marta-Simões, M.S. \\ Ana Laura Mendes, M.S. \\ Cláudia Ferreira, M.S., Ph.D \\ Inês Almeida Trindade, M.S., Ph.D. Student
}

Cognitive and Behavioural Center for Research and Intervention, University of Coimbra

\begin{abstract}
The inability of recalling warm and safe memories with parents and close relatives has been often associated in literature with a negative and judgmental sense of self, and a higher proneness to experience feelings of inferiority, inadequacy, and defectiveness. Thus, intending to deal with self-judgment and inferiority, individuals may become submissive as a way of compensating one's negative emotional states with other's positive attention and desirability. However, both early negative affiliative memories and submissiveness are associated with higher vulnerability to psychopathology, namely depression. Using a sample of 338 young women, the present study intended to examine the association between early affiliative memories and depressive symptomatology, and the mediator roles of self-judgment and submissive attitudes and behaviours on this association, through a path analysis. The tested model provided an excellent fit to the data, accounting for $41 \%$ of the depressive symptomatology's variance. Results revealed a direct effect of early affiliative memories on depressive symptomatology; and also on
\end{abstract}


self-judgement and submissiveness, explaining $28 \%$ and $23 \%$ of their variances, respectively. Moreover, part of these memories' effect on depressive symptomatology was explained by self-judgment and submissiveness, which seems to suggest that submissiveness, although used to compensate feelings of inferiority and a judging attitude towards the self, may be a maladaptive strategy due to its positive association with depressive symptoms. This study's findings appear to emphasize the relevance of targeting submissiveness, especially in the context of a scarce recall of early affiliative experiences, when approaching women's depressive symptomatology on mental health promotion programs. 